\title{
Scallop Theorem and Swimming at the Mesoscale
}

\author{
M. Hubert $\odot,{ }^{1, *}$ O. Trosman $\odot,{ }^{1}$ Y. Collard $\odot,{ }^{2}$ A. Sukhov $\odot,{ }^{3}$ J. Harting $\odot{ }^{3,4}$ N. Vandewalle $\odot,{ }^{2}$ and A.-S. Smith $\oplus^{1,5, \dagger}$ \\ ${ }^{1}$ PULS Group, Department of Physics and Interdisciplinary Center for Nanostructured Films, \\ FAU Erlangen-Nürnberg, 91058 Erlangen, Germany \\ ${ }^{2}$ GRASP, Research unit CESAM, Institute of Physics B5a, Université de Liège, 4000 Liège, Belgium \\ ${ }^{3}$ Helmholtz Institute Erlangen-Nürnberg for Renewable Energy (IEK-11), Forschungszentrum Jülich, 90429 Nürnberg, Germany \\ ${ }^{4}$ Department of Chemical and Biological Engineering and Department of Physics, \\ FAU Erlangen-Nürnberg, 90429 Nürnberg, Germany \\ ${ }^{5}$ Group for Computational Life Sciences, Division of Physical Chemistry, Ruđer Boskovic Institute, 10000 Zagreb, Croatia
}

(Received 18 August 2020; accepted 28 April 2021; published 2 June 2021)

\begin{abstract}
By comparing theoretical modeling, simulations, and experiments, we show that there exists a swimming regime at low Reynolds numbers solely driven by the inertia of the swimmer itself. This is demonstrated by considering a dumbbell with an asymmetry in coasting time in its two spheres. Despite deforming in a reciprocal fashion, the dumbbell swims by generating a nonreciprocal Stokesian flow, which arises from the asymmetry in coasting times. This asymmetry acts as a second degree of freedom, which allows the scallop theorem to be fulfilled at the mesoscopic scale.
\end{abstract}

DOI: 10.1103/PhysRevLett.126.224501

The time reversibility and linearity of the Stokes equation require microswimmers to deform in a nonreciprocal fashion in order to swim, a rule known as the "scallop theorem" [1]. Many strategies in the Stokesian regime, based on the periodic deformation of at least two degrees of freedom for successful propulsion, have been intensively investigated in the past decades [2-10]. This research has provided a fundamental understanding of the underlying dynamics as reflected by the emergence of several technological applications [11-16].

A natural way to break down the scallop theorem is by introducing inertia. This is commonly achieved by the inertial dynamics of the fluid [17,18], here characterized by the Reynolds number $\operatorname{Re}_{\mathrm{f}}=\rho_{f} L \bar{U} / \eta\left(\rho_{f}\right.$ and $\eta$ being the fluid density and viscosity, $L$ the swimmer body length, and $\bar{U}$ the average swimming speed). For example, the inclusion of inertia in the dynamics can be achieved by using steady streaming [19-21], vortex generation [22], or turbulent flows [23]. The possibilities for the exploitation of the swimmer's own inertia were first suggested a decade ago by Gonzalez-Rodriguez and Lauga [24], opening the debate on and additional investigations of swimming in this regime $[20,21,25]$. Interestingly, recent experiments and simulations have shown that mesoscopic structures, i.e., $100 \mu \mathrm{m}$ to $1 \mathrm{~cm}$ in scale, display coasting effects [26-28] while generating fluid flows with a time-reversible behavior $[29,30]$. Those observations point to the possible existence of a swimming regime at low $\mathrm{Re}_{\mathrm{f}}$ where the inertia of the socalled mesoswimmer dominates and generates the motion, a hypothesis that warrants further investigation.

A minimal mesoswimmer that can verify this hypothesis is an asymmetric dumbbell consisting of two different interacting beads driven in a force-free manner (Fig. 1). The Reynolds number $\operatorname{Re}_{\mathrm{s}}$ of such a swimmer is set by its bead density $\rho_{s}$, its bead size $a$, and its beating frequency $\omega$ such that $\operatorname{Re}_{\mathrm{s}}=\rho_{s} a^{2} \omega / \eta$. This design possesses only one internal degree of freedom, which leads to a reciprocal deformation, and therefore it cannot swim without the help of inertia $[19,20,24]$. Assuming that the Reynolds number of the fluid is $\operatorname{Re}_{\mathrm{f}} \ll 1$ and of the swimmer $\operatorname{Re}_{\mathrm{s}} \sim 1$, the flow should be dominated by the fluid viscosity while the propulsion mechanism should be related to the coasting time of the swimmer, which we define as $\tau=m /(6 \pi \eta a)$, with $m$ being the bead mass. In this case, the swimmer should achieve propulsion and fulfil the requirement of the scallop theorem by relying on an asymmetry in coasting times of the constitutive beads.

To elaborate on this idea, we first build an analytic theory that relates the swimming velocity and coasting times. We successfully compare the model to experiments and lattice Boltzmann simulations in which no assumptions are imposed, thereby verifying the hypothesis that there is a swimming regime in which the inertial effects in the swimming dynamics can be separated and the swimmer coasting time harnessed for propulsion.

Our modeling efforts revolve around a dumbbell [Fig. 1(a)] that consists of two submerged beads of mass $m_{i}$ and radii $a_{i}$. The beads are linked by a linear spring with stiffness $k$ and natural length $L$, capturing, within the harmonic approximation $\vec{G}_{i, j}=-k\left(\left|\vec{x}_{i}-\vec{x}_{j}\right|-L\right)$, possible direct interactions between beads. The external forcing $\vec{F}_{i}$ is a sinusoidal force applied to each bead with the same intensity $F$ and frequency $\omega$ in opposite directions to satisfy the force-free condition. The 


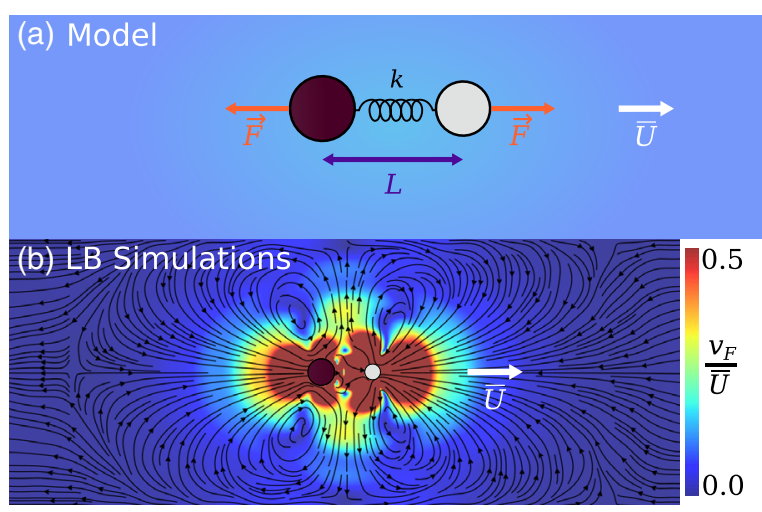

(c) Experiments

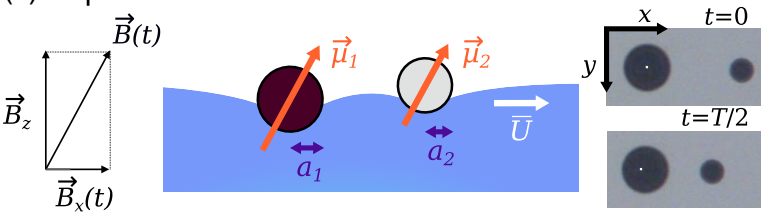

FIG. 1. Asymmetric swimming dumbbell. (a) The model assumes two spheres of density $\rho_{s}$ with radii $a_{1}$ and $a_{2}$ at a distance $L$ connected by a linear spring with constant $k$. The device is submerged in a fluid of viscosity $\eta$ and driven by sinusoidal forces of the same amplitude $F$ and frequency $\omega$ acting in opposite directions. (b) An equivalent system is addressed by lattice Boltzmann simulations $\left(a_{1}=8, \quad a_{2}=5, \quad \eta=1 / 6\right.$, $k=1 / 50, L=28, \rho_{f}=1$, and $\rho_{s}=8$, all expressed in lattice units or l.u.). The simulation box is discretized by $400 \times 160 \times$ 160 lattice nodes. The background shows the flow field averaged over one cycle of the external sinusoidal forcing $(F=0.1$, $\omega=1.57 \times 10^{-3}$ ). (c) A magnetocapillary dumbbell made of two ferromagnetic beads (magnetic moments $\vec{\mu}_{i}$, diameter $2 a_{i} \in(397,500,793) \mu \mathrm{m}$, and density $\left.7830 \mathrm{~kg} / \mathrm{m}^{3}\right)$ pinned at the water-air interface. The two beads' separation $L$ is about $1400 \mu \mathrm{m}$ and set by the balance of forces originating from capillary attraction and magnetic dipole repulsion. The device is driven by an external magnetic field $\vec{B}(t)=B_{z} \vec{e}_{z}+$ $\left[B_{0}+b \sin (\omega t)\right] \vec{e}_{x}$, which induces small oscillations of the beads. Snapshots are from the beginning of the cycle and halfway through.

swimming dynamics of this object is studied using the following equation of motion:

$$
\frac{\partial \boldsymbol{x}}{\partial t}=\hat{M}(\boldsymbol{x})\left[\boldsymbol{F}(t)+\boldsymbol{G}(\boldsymbol{x})-\hat{m} \frac{\partial^{2} \boldsymbol{x}}{\partial t^{2}}\right],
$$

where bold symbols account for concatenated vectors, e.g., $\boldsymbol{x}=\left(\vec{x}_{1}, \vec{x}_{2}\right)$. In this equation, we assume low $\mathrm{Re}_{\mathrm{f}}$ dynamics by using the mobility matrix $\hat{M}(\boldsymbol{x})$. This matrix models hydrodynamic interactions with the Stokes drag (diagonal elements) and the Oseen tensor (off diagonal elements). The inertia of the beads is explicitly taken into account by a force $-\hat{m}\left(\partial^{2} \boldsymbol{x} / \partial t^{2}\right)$. The matrix $\hat{m}$ has $m_{1}$ and $m_{2}$ in its diagonal elements.

This equation is solved using a perturbative scheme [7] (see also [31], Sec. I. A.). Assuming $F /\left(k a_{i}\right) \ll 1$ and $a_{i} / L \ll 1$, one obtains the period-averaged swimming speed

$$
\begin{aligned}
\bar{U}^{F}= & \frac{3 F^{2} \omega_{0}^{4}}{2 k^{2} \bar{\theta}^{2}} \frac{a_{1}^{2} a_{2}^{2}}{\left(a_{1}+a_{2}\right)^{3} L^{2}} \\
& \times \frac{\omega\left(\theta_{1}-\theta_{2}\right)}{\left[\left(\omega_{0}^{2}-\omega^{2}+\frac{\omega^{2}}{\theta_{1} \theta_{2}}\right)^{2}+\left(\frac{\omega_{0}^{2}}{\theta}-\frac{\omega^{2}}{\theta_{1}}-\frac{\omega^{2}}{\theta_{2}}\right)^{2}\right]},
\end{aligned}
$$

where $\theta_{i}=m_{i} \omega /\left(6 \pi \eta a_{i}\right)=\tau_{i} \omega$ is the ratio of the coasting time to the external forcing period, $\bar{\theta}=\left(m_{1}+m_{2}\right) \omega /$ $\left[6 \pi \eta\left(a_{1}+a_{2}\right)\right]$ is the swimmer coasting time, and $\omega_{0}^{2}=k\left(m_{1}+m_{2}\right) /\left(m_{1} m_{2}\right)$. The speed is expressed along the vector pointing from 1 to 2 . The solution for arbitrary separation (within the limit of the validity of the Oseen tensor) is provided in [31] (Sec. I. A.). The superscript $F$ in Eq. (2) refers to a force-based approach $[6,7,37]$ where the stroke of the beads is known only a posteriori.

Alternatively, one can impose a stroke a priori $[24,25]$ and calculate the swimming velocity $\bar{U}^{S}[2,3]$. Now $\boldsymbol{G}(\boldsymbol{x})$ is removed from Eq. (1). Assuming $a_{i} / L \ll 1$ and a stroke $\left|\vec{x}_{2}(t)-\vec{x}_{1}(t)\right|=L+d \sin (\omega t)$ of small amplitude $d / L \ll 1$ (see [31], Sec. I. B.), one obtains

$$
\bar{U}^{S}=\frac{3 d^{2}}{2} \frac{a_{1}^{2} a_{2}^{2}}{\left(a_{1}+a_{2}\right)^{3} L^{2}} \frac{\omega\left(\theta_{1}-\theta_{2}\right)}{1+\bar{\theta}^{2}} .
$$

Notably, there is a unique mapping between the strokebased and force-based approaches (see [31], Sec. I. C.), a result that is not recovered by comparisons with alternative calculations $[24,25]$.

In both force-based and stroke-based protocols, the analytical model described with Eq. (1) predicts a translation of the device in the direction of the beads with the smallest coasting time. This result may be sensitive to the $a_{i} / L$ conditions, as it can be seen in [31] where $\bar{U}^{F}$ is calculated without approximation beyond the use of the Oseen tensor.

One can relax the assumptions made on the hydrodynamic flows and study the asymmetric dumbbell with lattice Boltzmann (LB) simulations [Fig. 1(b)] [27,38,39]. This algorithm solves a discrete version of the Boltzmann equation and recovers solutions of the Navier-Stokes equations in the limit of low Mach and low Knudsen numbers. Additionally, LB simulations are not limited in the Reynolds number and account for all inertial effects in the dynamics of the fluid. For the bead dynamics, a leapfrog algorithm is used to solve Newton's equation of motion. The beads are discretized on the fluid lattice, and their dynamics is coupled to the fluid by a midgrid bounceback boundary condition $[27,31,40,41]$. As such, both the fluid and the spring-connected beads are simulated without any dynamical assumptions (see [31], Sec. II. A.). Therefore, a favorable comparison with the theoretical model would validate the assumption of separating the 
fluid and beads' inertia and neglecting the former. For further comparison, we choose the numerical parameters to recover the expected Reynolds numbers of the beads and the fluid. We first confirm that there is no net flow responsible for the swimmer's displacement and that a symmetric dumbbell does not swim. Finally, we show that a reciprocal deformation of an asymmetric pair results in a translational motion of the device in the direction of the small bead, as predicted by the theory.

Finally, we perform experiments using the magnetocapillary swimmers (see [31], Sec. II. B.) established previously [28-30,42] [Fig. 1(c)]. Those experiments are taking place in the underdamped limit [26], while the flows generated are time-reversible and therefore in the Stokesian regime [43]. In this limit, this assembly behaves as a Golestanian swimmer $[3,28]$, a result that stems from the fact that the equation of motion [Eq. (1)] can capture the swimmer dynamics at the zeroth order (i.e., as if placed in the bulk), despite the presence of the interface $[44,45]$, up to a scaling factor in the Stokes drag [26]. As a consequence, magnetocapillary dumbbells are perfect candidates for investigating the swimmer-inertia-driven dynamics.

In short, paramagnetic beads with a radius of 397,500 or $793 \mu \mathrm{m}$ are deposited on an air-water interface. Thanks to surface tension and the wetting line being pinned at the beads' surface, the beads are restricted to moving along the fluid interface, implying that gravity can be neglected in the dynamics. When placed in a magnetic field $B_{z}$ perpendicular to the interface, their capillary attraction is balanced by magnetic dipole repulsion [29]. Imposing a small oscillating field $\left[B_{0}+b \sin (\omega t)\right]$ in the direction parallel to the interface induces oscillations in the relative distance between the beads. The homogeneity of $B_{z}$ and the flatness of the interface away from the beads ensure forcefree conditions at all times. Measures are also taken to ensure that propulsion arises solely because of hydrodynamic interactions and not because of parasite effects, as described in [31]. Consequently, symmetric dumbbells with two identical beads show no self-propulsion. However, a linear and common displacement of the pair of beads is observed for two beads of different sizes. The swimmer moves toward the small bead, as shown in Fig. 2(a) (see also [31], SM Movie 1), in agreement with simulations and theoretical predictions. The swimmer is typically slow, reaching speeds up to $15 \mu \mathrm{m} / \mathrm{s}$, i.e., $4 \times 10^{-3} L / T$ body-length $L$ per period $T$, which gives a flow dominated by viscous drag instead of inertia, as quantified with $\mathrm{Re}_{\mathrm{f}} \sim 10^{-2}$. Similar speeds and Reynolds numbers were obtained in previous experiments involving the linear 3-bead swimmer [28].

In order to understand the role of the swimmer inertia, we analyze the frequency response of the swimming speed of the dumbbell [Figs. 2(b) and 2(c)] using all three approaches, whereby the parameters of the simulations are adjusted to recover the experimental swimmer geometry. In experiments, the investigated frequency range corresponds approximately to $\operatorname{Re}_{\mathrm{s}} \sim 1.5$ up to $\operatorname{Re}_{\mathrm{s}} \sim 15$, with the radius of the small bead used as the characteristic length [Fig. 2(b)]. This is matched in simulations where $\mathrm{Re}_{\mathrm{s}}$
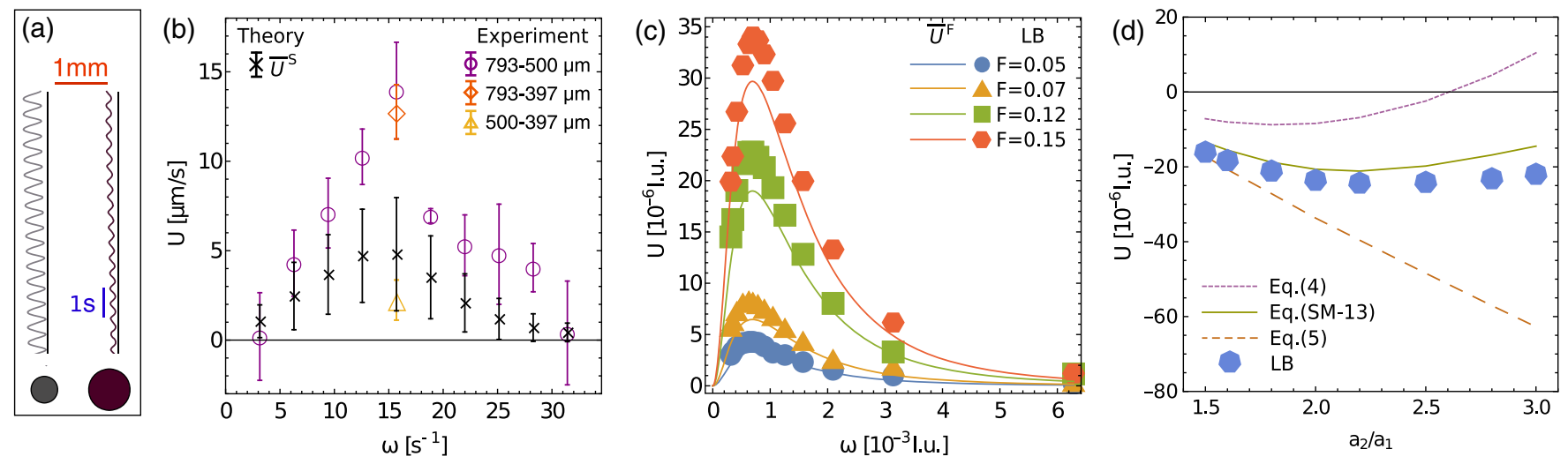

FIG. 2. Swimming dynamics of an asymmetric dumbbell-comparison of LB simulations and experiments to the analytic model. (a) Sinusoidal trajectory of the beads $(500$ and $793 \mu \mathrm{m})$ subject to the driving $B_{z}=5.6 \mathrm{mT}, B_{0}=0.7 \mathrm{mT}, b=0.35 \mathrm{mT}$, and $\omega=12.57 \mathrm{~Hz}$. Vertical lines are guides to the eye. (b) Average swimming speed of the asymmetric dumbbell as a function of the magnetic field frequency $\omega$. Amplitudes of the field are as in (a). Error bars account for the variance $( \pm \sigma)$ between five independent experiments. Theory using Eq. (3) is shown in black for the 500-793 $\mu \mathrm{m}$ beads' combination, with the error bars propagated from experimental uncertainty. (c) Average swimming speed of the asymmetric dumbbell as a function of the frequency from LB simulations (symbols) and the analytic model based on Eq. (SM-13) in the Supplemental Material (SM) [31] (curves) for different values of the driving force $F$ (no fitting). Parameters are the same as in Fig. 1 except that $k=1 / 200$. (d) Average swimming speed of the dumbbell when increasing the radii asymmetry between fore and aft. The points are obtained using LB simulations for $\omega=1.26 \times 10^{-3} 1 . u$. and $k=1 / 200$ l.u. The value of $a_{2}$ is allowed to change, while $a_{1}$ is fixed to 3 1.u.. The green line is obtained based on Eq. (SM-13) using the parameters of the simulation. For the curves accounting for Eqs. (4) and (5), the amplitudes of oscillations have been extracted from the simulated trajectories. 
ranges from 0.38 to 7.54 for the frequencies considered [Fig. 2(c)], while $\mathrm{Re}_{\mathrm{f}}$ remains small at $\mathrm{Re}_{\mathrm{f}}<2 \times 10^{-3}$.

For low frequencies corresponding to $\operatorname{Re}_{\mathrm{s}} \ll 1$, with $\operatorname{Re}_{\mathrm{f}} \ll 1$, the asymmetric swimmer obeys the usually encountered Stokesian scallop theorem for microswimmers [1]. Consequently, the dumbbell swims inefficiently. A vanishing swimming speed is also observed at high frequencies in all approaches as the amplitude of oscillation decreases. The intermediate frequencies are characterized by a broad peak in the dumbbell speed. Following the analytic model, this maximum should be associated with the mechanical resonance of the dumbbell. Specifically, Eq. (2) possesses an optimal swimming frequency close to $\omega_{0}$, a signature of the influence of the swimmer inertia. This maximum is thus by nature different from the optimum frequency occurring for purely Stokesian dynamics [6,37]. In experiments, the maximum appears at a frequency of around $15 \mathrm{~s}^{-1}$, which corresponds to the characteristic mechanical resonance identified previously $[26,28]$. In simulations, it occurs around $\mathrm{Re}_{\mathrm{s}}=1.5$, which corresponds well to $\omega_{0} \sim 1.22 \times 10^{-3}$ l.u.

Finally, we compare the analytic model directly to the experiments [Fig. 2(b)] and simulations [Fig. 2(c)]. Starting with the simulations, rather than using Eq. (2), we use Eq. (SM 13) (no restriction on $a_{i} / L$ ) due to the proximity of the beads in the simulations. With no fitting parameters, the agreement is excellent, with the error not exceeding $10 \%$. The strongest deviations are found around the peak velocity, where the nonzero fluid inertia may play a small role $[18,20,21]$. Furthermore, from reading out the stroke amplitude obtained in experiments, the measured velocities can be compared to the model using Eq. (3) [see Fig. 2(b)]. Once again, a very good agreement is obtained with some differences in speed amplitudes at higher frequencies. This deviation could be attributed to the presence of the interface and the nonlinearity of the magnetocapillary potential, which are not captured by the model.

We also analyze the evolution of the swimming speed as a function of the asymmetry ratio between the size of the spheres, namely $a_{2} / a_{1}$ in Fig. 2(d). This evaluation allows us to compare our results to a previously developed strokebased approach reported in [24]. Relying on the reciprocal theorem, the authors [24] calculate the velocity of a swimmer with beads of identical densities and also for a vanishing $\operatorname{Re}_{s}$. This leads, in our notation, to the following swimming speed:

$$
\bar{U}^{\star}=\frac{\rho_{b} \omega^{2} d^{2}}{6 \eta L^{2}} \frac{a_{1} a_{2}\left(a_{1}^{2}-a_{2}^{2}\right)\left(3 a_{1} a_{2}-a_{1}^{2}-a_{2}^{2}\right)}{\left(a_{1}+a_{2}\right)^{3}} .
$$

This result is different from our own stroke-based theory, which is obtained by resolution of Eq. (1). If cast in the same limit, Eq. (3) reads

$$
\begin{aligned}
\bar{U}_{0}^{S} & =\lim _{\operatorname{Re}_{s} \rightarrow 0} \bar{U}^{S}=\frac{\rho_{b} \omega^{2} d^{2}}{3 \eta L^{2}} \frac{a_{1}^{2} a_{2}^{2}\left(a_{1}^{2}-a_{2}^{2}\right)}{\left(a_{1}+a_{2}\right)^{3}}, \\
& =\bar{U}^{\star} \frac{2 a_{1} a_{2}}{\left(3 a_{1} a_{2}-a_{1}^{2}-a_{2}^{2}\right)} .
\end{aligned}
$$

Both models are compared to the LB simulations. At the same level of theory, clearly both models significantly deviate from the LB simulations. Indeed, Eq. (5) systematically overestimates the negative swimming velocity but leads to a good approximation at low $a_{2} / a_{1}$, where the inertia is small and the low $\operatorname{Re}_{s}$ approximation is valid. However, Eq. (4) demonstrates a reversal in the swimming direction at $a_{2} / a_{1}=(3+\sqrt{5}) / 2$ that is not seen in the LB simulations or in our approach. Furthermore, if we compare the LB simulations with the force-based approach (for which mapping onto the stroke calculations was demonstrated already), we can apply our model without any fitting parameter, in which case the LB results are recovered nicely [Fig. 2(d)]. Notably, our swimmer dynamics is comparable to that of a neutrally buoyant deforming dumbbell at low $\operatorname{Re}_{f}[20,21]$, where, indeed, self-propulsion in the direction of the small bead was also found.

Those comparisons not only vindicate the theoretical model but also testify to the existence of a mesoscopic swimming regime where propulsion is driven by the inertia of the device while keeping a low $\mathrm{Re}_{\mathrm{f}}$. Self-propulsion of mesoswimmers relies on $\operatorname{Re}_{\mathrm{s}}>1$, which points to the significant role of the inertia of the beads. However, as demonstrated by the behavior of the symmetric design, inertia alone is not able to propel with a reciprocal deformation. Indeed, swimming necessitates the asymmetry of the design. Under the application of forces, beads accelerate and decelerate at a different rate as soon as $\theta_{i} \neq 0$ [24]. A direct consequence of this asymmetric response is to induce a phase shift (Fig. 3) in the oscillation of the beads measured within the laboratory frame (see [31], Sec. I. C.). This is captured by an ellipse in the configuration space of the dumbbell spanned by the coordinates $\vec{x}_{1}$, and $\vec{x}_{2}$ of the two oscillating beads. As a consequence of this phase difference, the velocity of the beads with respect to the fluid is not time-reversible even though the swimmer deforms in a reciprocal fashion. It is worth noticing that this phase shift is constant with respect to time as soon as the transient dynamics of the beads is over. This has been verified in the
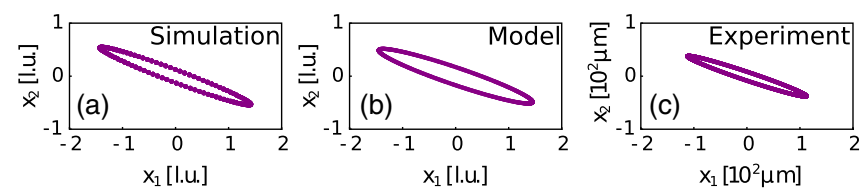

FIG. 3. Configuration space of the dumbbell. The ellipse results from the phase shift in the oscillations of the beads $x_{1}(t)$ and $x_{2}(t)$ around the swimmer geometric center. (a) LB simulations, (b) corresponding analytics $\left(\omega=1.57 \times 10^{-3}\right)$, and (c) experiments $\left(\omega=15.7 \mathrm{~s}^{-1}, 500-793 \mu \mathrm{m}\right)$ 
numerics and in the experiments and also validated by the theory [31]. In short, the two configurational degrees of freedom required by the scallop theorem are located in the individual oscillations of the beads, which are naturally out of phase thanks to the difference of the beads' coasting time.

The phase shift has two consequences. First, it implies that despite having a force-free swimmer, the instantaneous flow field generated by the swimmer can have a monopolar component (see [31], Sec. I. D.). Nevertheless, the timeaveraged flow is dipolar [Fig. 1(b)], and the swimmer can be described as a puller in the investigated range of parameters. Notably, similar flow fields are also generated in neutrally buoyant deforming dumbbells $[20,21]$, showing that the small inertia of the fluid does not qualitatively change the swimmer's behavior.

Second, knowing the phase shift in the individual oscillations also allows us to cast the expression of the swimming speed into

$$
\bar{U} \propto A_{1} A_{2} \sin \Delta \phi,
$$

where $A_{i}$ are the amplitudes of oscillation of the beads (see [31], Secs. I. B.-I. C.). This result states that the dumbbell naturally fulfills the scallop theorem by relying on the beads' nonzero coasting time and its asymmetry.

In conclusion, this Letter rephrases the scallop theorem for mesoswimmers and hence establishes the physical framework for self-propulsion in the vanishing $\operatorname{Re}_{f}$ yet finite $\mathrm{Re}_{s}$ regime. Contrary to previous works, we show that the theorem can be recast on a mesoscopic scale thanks to the nonreciprocal flow field generated by the two spheres that originates from the competing timescales of the dynamics, namely the coasting times and external forcing period. We are able to present this result by successfully bringing together theoretical modeling, numerical simulations, and experiments. The analysis performed here thus shows that the transition from microswimmers to mesoswimmers may occur through a delicate balance of viscous damping and inertial relaxation. At higher Reynolds numbers, naturally, the inertia of the fluid will couple to the coasting of the swimmer and dominate the dynamics $[20,21]$. The analysis provided here may help explain the emergence of this complex interplay.

We thank S. Ziegler and G. Grosjean for insightful discussions. The funding was provided by the Deutsche Forschungsgemeinschaft (DFG, German Research Foundation)—Project-ID 416229255-SFB 1411 and the priority program SPP1726 (grant HA4382/5-1), as well by the FNRS grant PDR T.0129.18. Simulations were performed at the Jülich Supercomputing Centre, the High Performance Computing Center Stuttgart, and the Regionales Rechenzentrum Erlangen.

M. H., O.T., Y.C., and A.S. contributed equally to this work.
*Corresponding author. maxime.hubert@fau.de ${ }^{\dagger}$ Corresponding author. ana-suncana.smith@fau.de or asmith@irb.hr

[1] E. M. Purcell, Life at low Reynolds number, Am. J. Phys. 45, 3 (1977).

[2] A. Najafi and R. Golestanian, Simple swimmer at low Reynolds number: Three linked spheres, Phys. Rev. E 69, 062901 (2004).

[3] R. Golestanian and A. Ajdari, Analytic results for the threesphere swimmer at low Reynolds number, Phys. Rev. E 77, 036308 (2008).

[4] J. E. Avron, O. Kenneth, and D. H. Oaknin, Pushmepullyou: An efficient micro-swimmer, New J. Phys. 7, 234 (2005).

[5] F. Y. Ogrin, P. G. Petrov, and C. P. Winlove, Ferromagnetic Microswimmers, Phys. Rev. Lett. 100, 218102 (2008).

[6] J. Pande and A.-s. Smith, Forces and shapes as determinants of micro-swimming: Effect on synchronisation and the utilisation of drag, Soft Matter 11, 2364 (2015).

[7] S. Ziegler, M. Hubert, N. Vandewalle, J. Harting, and A.-S. Smith, A general perturbative approach for bead-based microswimmers reveals rich self-propulsion phenomena, New J. Phys. 21, 113017 (2019).

[8] R. Dreyfus, J. Baudry, M. L. Roper, M. Fermigier, H. A. Stone, and J. Bibette, Microscopic artificial swimmers, Nature (London) 437, 862 (2005).

[9] J. K. Hamilton, P. G. Petrov, C.P. Winlove, A. D. Gilbert, M. T. Bryan, and F. Y. Ogrin, Magnetically controlled ferromagnetic swimmers, Sci. Rep. 7, 44142 (2017).

[10] P. Tierno, R. Golestanian, I. Pagonabarraga, and F. Sagués, Magnetically actuated colloidal microswimmers, J. Phys. Chem. B 112, 16525 (2008).

[11] S. Leulmi, X. Chauchet, M. Morcrette, G. Ortiz, H. Joisten, P. Sabon, T. Livache, Y. Hou, M. Carrière, S. Lequien, and B. Dieny, Triggering the apoptosis of targeted human renal cancer cells by the vibration of anisotropic magnetic particles attached to the cell membrane, Nanoscale 7, 15904 (2015).

[12] W. Gao, B. E.-F. de Ávila, L. Zhang, and J. Wang, Targeting and isolation of cancer cells using micro/nanomotors, Adv. Drug Delivery Rev. 125, 94 (2018).

[13] M. Medina-Sánchez, L. Schwarz, A. K. Meyer, F. Hebenstreit, and O. G. Schmidt, Cellular cargo delivery: Toward assisted fertilization by sperm-carrying micromotors, Nano Lett. 16, 555 (2016).

[14] H. Xu, M. Medina-Sánchez, V. Magdanz, L. Schwarz, F. Hebenstreit, and O. G. Schmidt, Sperm-hybrid micromotor for targeted drug delivery, ACS Nano 12, 327 (2018).

[15] J. Orozco, G. Pan, S. Sattayasamitsathit, M. Galarnyk, and J. Wang, Micromotors to capture and destroy anthrax simulant spores, Analyst 140, 1421 (2015).

[16] S. Campuzano, J. Orozco, D. Kagan, M. Guix, W. Gao, S. Sattayasamitsathit, J. C. Claussen, A. Merkoçi, and J. Wang, Bacterial isolation by lectin-modified microengines, Nano Lett. 12, 396 (2012).

[17] E. Lauga, Continuous breakdown of Purcell's scallop theorem with inertia, Phys. Fluids 19, 061703 (2007). 
[18] D. Klotsa, As above, so below, and also in between: Mesoscale active matter in fluids, Soft Matter 15, 8946 (2019).

[19] D. Klotsa, K. A. Baldwin, R. J. A. Hill, R. M. Bowley, and M. R. Swift, Propulsion of a Two-Sphere Swimmer, Phys. Rev. Lett. 115, 248102 (2015).

[20] T. Dombrowski, S. K. Jones, G. Katsikis, A. P. S. Bhalla, B. E. Griffith, and D. Klotsa, Transition in swimming direction in a model self-propelled inertial swimmer, Phys. Rev. Fluids 4, 021101(R) (2019).

[21] T. Dombrowski and D. Klotsa, Kinematics of a simple reciprocal model swimmer at intermediate Reynolds numbers, Phys. Rev. Fluids 5, 063103 (2020).

[22] D. L. Hu, B. Chan, and J. W. M. Bush, The hydrodynamics of water strider locomotion, Nature (London) 424, 663 (2003).

[23] M. Gazzola, M. Argentina, and L. Mahadevan, Scaling macroscopic aquatic locomotion, Nat. Phys. 10, 758 (2014).

[24] D. Gonzalez-Rodriguez and E. Lauga, Reciprocal locomotion of dense swimmers in Stokes flow, J. Phys. Condens. Matter 21, 204103 (2009).

[25] B. U. Felderhof, Effect of fluid inertia on the motion of a collinear swimmer, Phys. Rev. E 94, 063114 (2016).

[26] G. Lagubeau, G. Grosjean, A. Darras, G. Lumay, M. Hubert, and N. Vandewalle, Statics and dynamics of magnetocapillary bonds, Phys. Rev. E 93, 053117 (2016).

[27] A. Sukhov, S. Ziegler, Q. Xie, O. Trosman, J. Pande, G. Grosjean, M. Hubert, N. Vandewalle, A.-S. Smith, and J. Harting, Optimal motion of triangular magnetocapillary swimmers, J. Chem. Phys. 151, 124707 (2019).

[28] G. Grosjean, M. Hubert, G. Lagubeau, and N. Vandewalle, Realization of the Najafi-Golestanian microswimmer, Phys. Rev. E 94, 021101(R) (2016).

[29] G. Grosjean, G. Lagubeau, A. Darras, M. Hubert, G. Lumay, and N. Vandewalle, Remote control of self-assembled microswimmers, Sci. Rep. 5, 16035 (2015).

[30] G. Grosjean, M. Hubert, and N. Vandewalle, Magnetocapillary self-assemblies: Locomotion and micromanipulation along a liquid interface, Adv. Colloid Interface Sci. 255, 84 (2018).

[31] See Supplemental Material, which includes Refs. [32-36], at http://link.aps.org/supplemental/10.1103/PhysRevLett .126 .224501 for methods and additional discussions.
[32] Y. H. Qian, D. D'Humières, and P. Lallemand, Lattice BGK models for Navier-Stokes equation, Europhys. Lett. 17, 479 (1992).

[33] P. L. Bhatnagar, E. P. Gross, and M. Krook, A model for collision processes in gases. I. Small amplitude processes in charged and neutral one-component systems, Phys. Rev. 94, 511 (1954).

[34] A. J. C. Ladd, Numerical simulations of particulate suspensions via a discretized Boltzmann equation. Part 1. Theoretical foundation, J. Fluid Mech. 271, 285 (1994).

[35] F. Jansen and J. Harting, From bijels to Pickering emulsions: A lattice Boltzmann study, Phys. Rev. E 83, 046707 (2011).

[36] N. Vandewalle, L. Clermont, D. Terwagne, S. Dorbolo, E. Mersch, and G. Lumay, Symmetry breaking in a few-body system with magnetocapillary interactions, Phys. Rev. E 85, 041402 (2012).

[37] J. Pande, L. Merchant, T. Krüger, J. Harting, and A.-S. Smith, Setting the pace of microswimmers: When increasing viscosity speeds up self-propulsion, New J. Phys. 19, 053024 (2017).

[38] R. Benzi, S. Succi, and M. Vergassola, The lattice Boltzmann equation: Theory and applications, Phys. Rep. 222, 145 (1992).

[39] T. Krüger, H. Kusumaatmaja, A. Kuzmin, O. Shardt, G. Silva, and E. M. Viggen, The Lattice Boltzmann Method, Graduate Texts in Physics (Springer International Publishing, Cham, 2017).

[40] A. J. C. Ladd and R. Verberg, Lattice-Boltzmann simulations of particle-fluid suspensions, J. Stat. Phys. 104, 1191 (2001).

[41] J. Harting, H. J. Herrmann, and E. Ben-Naim, Anomalous distribution functions in sheared suspensions, Europhys. Lett. 83, 30001 (2008).

[42] Y. Collard, G. Grosjean, and N. Vandewalle, Magnetically powered metachronal waves induce locomotion in selfassemblies, Commun. Phys. 3, 112 (2020).

[43] G. Grosjean, M. Hubert, Y. Collard, A. Sukhov, J. Harting, A.-S. Smith, and N. Vandewalle, Capillary assemblies in a rotating magnetic field, Soft Matter 15, 9093 (2019).

[44] A. Dörr and S. Hardt, Driven particles at fluid interfaces acting as capillary dipoles, J. Fluid Mech. 770, 5 (2015).

[45] A. Dörr, S. Hardt, H. Masoud, and H. A. Stone, Drag and diffusion coefficients of a spherical particle attached to a fluid-fluid interface, J. Fluid Mech. 790, 607 (2016). 